\title{
ARMOS TurtleBot 1 Robotic Platform: Description, Kinematics and Odometric Navigation
}

\author{
Cristian F. PenagosB. ${ }^{\# 1}$, Luis A. Pacheco R.. ${ }^{\# 2}$ and Fredy H. Martínez S. ${ }^{\# 3}$ \\ ${ }^{\#}$ FacultadTecnológica, Universidad Distrital Francisco José de Caldas \\ Carrera 13 \#40-53, Bogotá D.C., Colombia \\ 1 cfpenagosb@correo.udistrital.edu.co \\ 2lapachecor@correo.udistrital.edu.co \\ ${ }^{3}$ fhmartinezs@udistrital.edu.co
}

\begin{abstract}
This paper describes the robotic platform ARMOS TurtleBot 1. The platform has been developed for research dedicated to care robotics. It is built on reconfigurable and scalable hardware, with the ability to adapt to specific tasks in motion planning and path planning, and operation both online and off-line. Here we present the development of its kinematic model, which in conjunction with odometric estimation drastically reduces errors in trajectories. The results of laboratory tests are shown in order to determine the accuracy in degrees of the turns and in centimetres of the advance using odometric estimation. The movements used in the tests correspond to those to be used in real environments.
\end{abstract}

Keyword - Differential wheeled robot, Kinematics, Odometric Navigation, Robotic platform

\section{INTRODUCTION}

Robotics is a major field of research, with a great deal of activity worldwide. Although it is born as a child of control and automation, its great possibilities in applications and the amount of intrinsic problems make it an autonomous field. Among the most desirable applications of robotics are those in which robots perform activities that support human beings $[1,2,3]$. These activities may be in industrial-level jobs in repetitive, dangerous, or complex and careful processes, in which case they work hand in hand with people (industrial robotics) [4], or they may be activities where the robot is in charge of people, for example, caring for sick people, children, or single people (service robotics) $[5,6,7]$.

Within this field of application of robotics there are a large number of specific tasks that must develop the robots. These tasks include: navigation in static or dynamic environments [8], manipulation and transport of objects from one place to another [9], attention to voice commands, emission of sounds or phrases, tracking of signals and/or objects [10], stable locomotion and rapid reaction to various external actions, among others. These isolated tasks could then be integrated to fulfil more complex tasks involving several of these methodologies, such as interaction with people. At the same time, given the complexity of the tasks of interaction with people, it is necessary to develop particular methodologies for each problem [11].

To investigate these problems there are a large number of commercial robot platforms [12, 13]. These platforms are characterized by their great capacity of movement, processing, communication and cost. In general, these platforms are not developed for a particular problem, but to try to solve as many problems as possible, hence their high level of sophistication and cost. A robotic platform limited to a specific problem turns out to be more discrete in resources and less expensive.

In the particular case of service robotics, a robotic platform must have specific characteristics that allow it to move in human environments on the one hand, and interact with human beings on the other [14]. The TurtleBot 1 robot platform was designed and built to be the mobile platform of an assistive robot (in the style of a mobile manipulator). As such, the platform must be able to move in flat indoor environments where GPS location is limited, but where it can use resources such as WiFi connectivity and power from electrical outlets.

The design of this platform followed some basic criteria regarding the resources required for the development of applied research. These criteria are reflected in the hardware and software design available in the robot. Given the displacement functions of the platform, it must be capable of executing path planning algorithms both geometric from external sensing of the environment, and reactive from local readings of the environment [15]. These two possibilities are present in the platform, but of particular interest in this paper are those strategies where the trajectories are defined by the analysis of the environment by means of a static camera in a zenithal position. 
One of the most important aspects related to the control of these strategies is the behaviour of the variables associated with the wheels of the robot. Control is achieved by manipulating the direct and reverse kinematic model of the robot. With the direct model, variables such as the position and orientation of the robot can be obtained from data read by the sensors. With the inverse model it is possible to configure the same variables to take the robot to a desired position or orientation. The first case is the one that interests us in this characterization of our platform, this method of location is known as odometric estimation or Odometric DeadReckoning $[16,17]$.

The problem with this method is that due to small errors in sensors and actuators, the location error of the robot cumulatively increases without limits $[18,19]$. Although the problem can be reduced by improving the model of the robot, this becomes an arduous task that makes the model only work on a specific robot, and for a limited time given the wear of its parts. Another way to attack the problem is to correct the position of the robot at regular intervals. This is among others the reason for interest in the method, since it produces good results on short distances with an acceptable model, and can be combined with simple absolute position schemes to reduce the cost of the global location scheme.

The following part of the paper is arranged in this way. Section 2 presents preliminary concepts and problem formulation. Section 3 illustrates the design profile and development methodology. Section 4 we present the preliminary results. And finally, in Section 5, we present our conclusions.

\section{Problem Formulation}

A differential wheeled robot is a robot with displacement from wheels (in general, but not strictly), whose movement depends on the difference in speed between the wheels on its two sides. In general it is based on two separately driven wheels placed on both sides of the robot body, but some other robot structures may have a similar displacement scheme even if they are not supported on wheels or are more than two. In this strategy of displacement the change of direction of advance is achieved by varying the relative speed of rotation from one side to the other, so it does not require an additional steering movement.

The kinematic model of a robot corresponds to the description of its mechanical movement without considering the forces involved in that movement. In the case of mobile robots such as our ARMOS TurtleBot 1, the purpose of the cinematic model is to represent the speed of the robot as a function of the speeds of its wheels, as well as the geometric parameters of the robot. According to Fig. 1, for a robot with $R$-radius wheels, the linear speed of each side wheel determines the average forward speed of the robot according to Equ. 1:

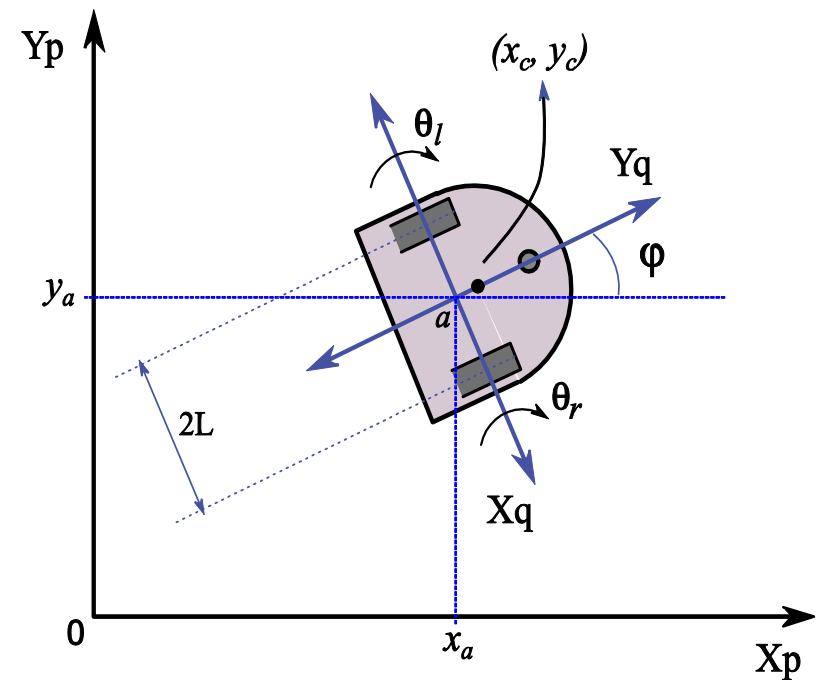

Fig. 1.Differential wheeled robot

$$
\left.v(t)=\frac{v_{r}(t)+v_{l}(t)}{2}=\frac{R}{2}\left(\theta_{r}(t)+\theta_{l} \dot{(} t\right)\right)(1)
$$

And its angular velocity is given by (Equ. 2):

$$
\left.\omega(t)=\frac{v_{r}(t)-v_{l}(t)}{2 L}=\frac{R}{2}\left(\theta_{r}(t)-\theta_{l} \dot{(} t\right)\right)(2)
$$


The speeds of the robot wheels can also be used to describe the speed of the geometric center of rotation of the robot (with respect to the robot, $(X q, Y q)$ ), which we will call point $a$ (Equ. 3):

Therefore (Equ. 4):

$$
\left\{\begin{array}{c}
x_{a} \dot{(}(t)=\frac{R}{2}\left(\theta_{r} \dot{(}(t)+\dot{\theta_{l}}(t)\right) \\
y_{a} \dot{(t)}=0 \\
\varphi \dot{(t)}=\omega(t)=\frac{R}{2 L}\left(\theta_{r} \dot{(t)}-\dot{\theta_{l}(t)}\right)
\end{array}\right.
$$

$$
\left[\begin{array}{c}
x_{a} \dot{(}(t) \\
y_{a}(t) \\
\varphi \dot{\varphi(t)}
\end{array}\right]=\left[\begin{array}{cc}
\frac{R}{2} & \frac{R}{2} \\
0 & 0 \\
\frac{R}{2 L} & -\frac{R}{2 L}
\end{array}\right]\left[\begin{array}{c}
\theta_{r}(t) \\
\theta_{l}(t)
\end{array}\right](4)
$$

With respect to the inertial frame of reference $(X p, Y p)$, equations can be written as (Equ. 5):

$$
\left[\begin{array}{c}
x_{a} \dot{(}(t) \\
y_{a}(t) \\
\varphi(t)
\end{array}\right]=\left[\begin{array}{cc}
\frac{R}{2} \cos \varphi & \frac{R}{2} \cos \varphi \\
\frac{R}{2} \sin \varphi & \frac{R}{2} \sin \varphi \\
\frac{R}{2 L} & -\frac{R}{2 L}
\end{array}\right]\left[\begin{array}{c}
\theta_{r}(t) \\
\theta_{l}(t)
\end{array}\right](5)
$$

This last equation represents the kinematic model of the robot. There is an alternative form of this model, in which the speeds of the robot are represented in terms of linear and angular speeds with respect to the robot (Equ. 6):

$$
\left[\begin{array}{c}
x_{a}(t) \\
y_{a}(t) \\
\varphi \dot{(}(t)
\end{array}\right]=\left[\begin{array}{cc}
\cos \varphi & \cos \varphi \\
\sin \varphi & 0 \\
0 & 1
\end{array}\right]\left[\begin{array}{c}
v(t) \\
\omega(t)
\end{array}\right](6)
$$

The goal is to find a suitable cinematic representation under these parameters for our ARMOS TurtleBot 1 (Fig. 1) robot and evaluate its performance. Although our robot does not have wheels, and in fact its displacement is achieved by means of four motors, the truth is that its characteristic of mobility coincides with the characteristic of a differential wheeled robot, reason why the model is valid. This is something that should also be verified in the performance evaluation.

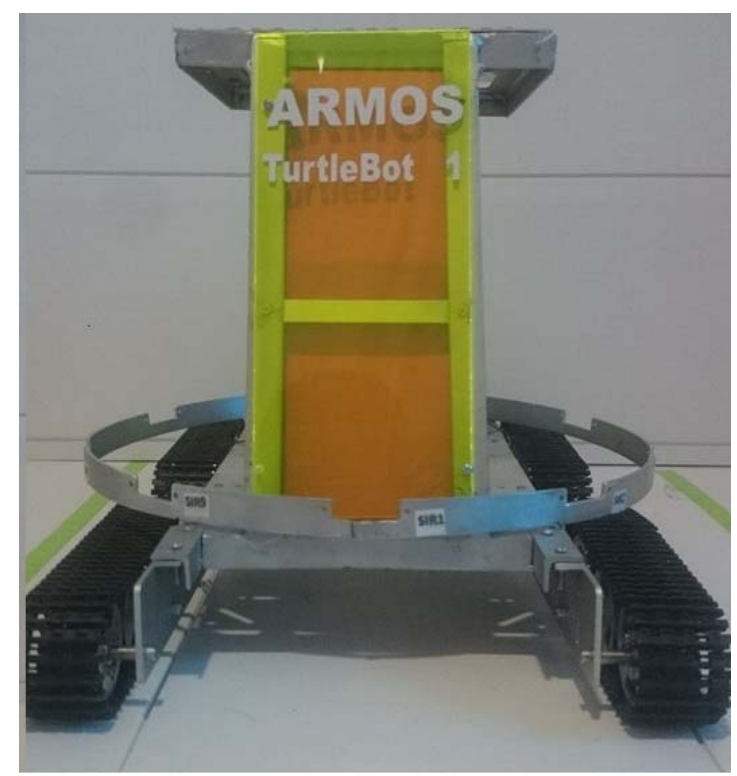

Fig. 2. ARMOS TurtleBot 1 robotic platform 


\section{Methodology}

The ARMOS TurtleBot 1 robot as sensors has nine IR sensors with a range of 0.1 to $0.8 \mathrm{~m}$ distributed around it with 40 degrees of separation one from the other, on a circumference. In the lower part the robot has a color sensor, and another inductive for the detection of distance to metals. The robot also has an accelerometer and a gyroscope. As actuators, the robot has four DC motors, one on each side. Each pair of motors (on each side of the robot) operate simultaneously to achieve the behaviour equivalent to a differential wheeled robot. Each motor has Hall Effect sensors for speed and rotation detection.

For the control of motors we use a drive with a nominal current higher than that of the motors at full load. For the handling of the sensors and motors we use two compatible Mbed cards (STM L432KC) that are communicated by $\mathrm{I}^{2} \mathrm{C}$ with the control unit. The control unit is implemented around a Dragonboard $410 \mathrm{C}$. The control unit also has a router for WiFi communication, and Bluetooth communication capability.

In a differential wheeled robot the behaviour of the wheels in the plane can be completely specified by means of four parameters: the point of contact of the wheels in the plane $(x, y)$, the angle $\theta$ between the vertical and a reference radius of the wheel which indicates how many times the wheel has turned, and an angle of orientation with respect to the $z$-axis. Making use of the transformations between reference systems we obtain the location in the Cartesian plane of our robot (Figs. 1 and 3).

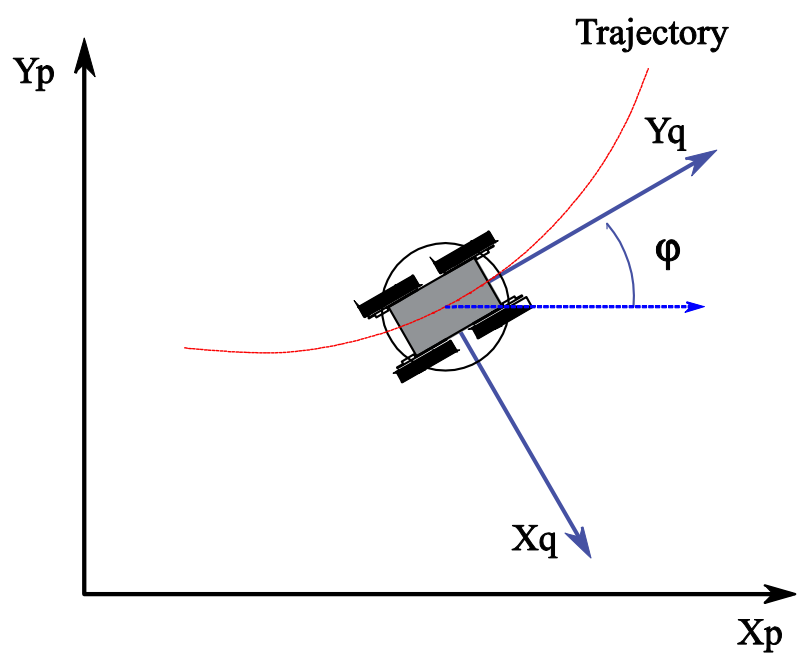

Fig. 3. Geometric description of the ARMOS TurtleBot 1 platform

For the determination of the odometric system we use Hall Effect sensors in each of the motors. These sensors send a pulse for each turn on the motor shaft (Fig. 4). Therefore, the pulse train of the sensor is directly proportional to the number of turns made by the drive wheel on the crawler (this is due to the ratio in the gearbox). With this data and the diameter of the drive wheel, it is possible to determine the variable distance travelled $d$ as $\left(d_{1}\right.$ distance travelled in one wheel turn,Equ. 7):

$$
d_{1}=\pi \times \text { diameter }
$$

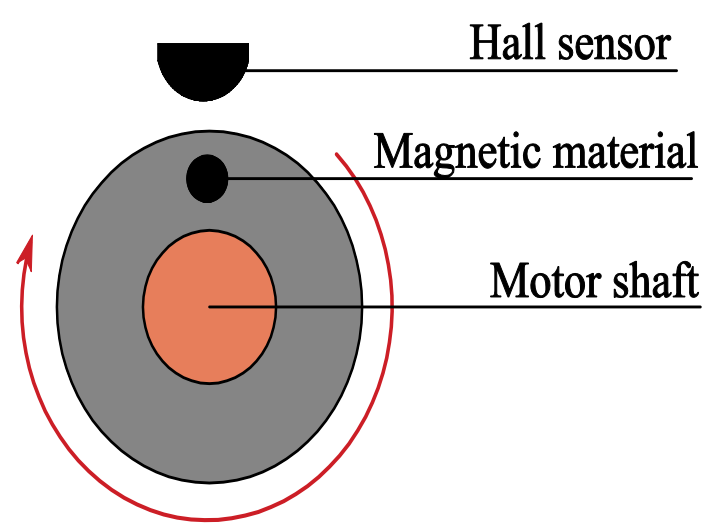

Fig. 4. Hall Effect sensor attached to the motor

For a wheel diameter of $48.30 \mathrm{~mm}$, then $d_{1}=151.74 \mathrm{~mm}$. Equ. 7 calculates the distance travelled in one turn of the wheel, i.e. for each turn the robot advances $15.17 \mathrm{~cm}$. If the sensor sends 76 pulses, then the resolution (maximum error expected in the measurement) in the measure of the advance is of (Equ. 8): 


$$
\frac{151.74}{76}=1.99 \mathrm{~mm}
$$

We design the test trajectories according to equations 5 and 6 . Due to the speed range in which the sensor can pick up turns without error, we set a Set Point of $10 \mathrm{~cm} / \mathrm{s}$ for the linear speed, and taking into account that the distance between wheels $L$ is $25 \mathrm{~cm}$, the angular speeds at which the motors must rotate to describe any trajectory are (Equs. 9 and 10):

$$
\begin{aligned}
\theta_{l}(t) & =\frac{10-\frac{25}{2} \omega(t)}{2.4} \mathrm{rad} / \mathrm{s} \\
\left.\theta_{r} \dot{(} t\right) & =\frac{10+\frac{25}{2} \omega(t)}{2.4} \mathrm{rad} / \mathrm{s}
\end{aligned}
$$

Where the radius of the wheel $R$ is $2.4 \mathrm{~cm}$.Under these conditions we test the robot with 90 degree turns and straight line advance to detect the cumulative error and distance restrictions of the kinematic model.

\section{RESUlts AND DISCUSSION}

\section{A. Turning tests at 90-degree angles}

In this test we program an algorithm in which the robot cyclically travels a square trajectory of $80 \mathrm{~cm}$ by 80 $\mathrm{cm}$, with the idea that the test path would be significantly larger than the robot (Fig. 5). During the route we register the movement of the robot on the environment, evaluating the errors that can arise in the turns. It is of particular interest to observe the performance during the turns of 90 degrees (corners).

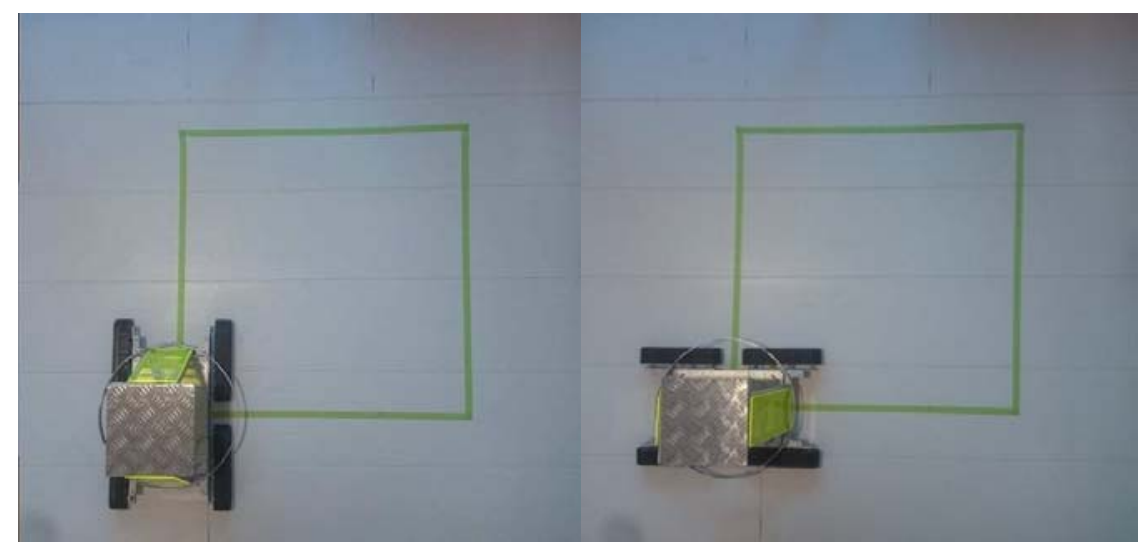

(a)

(b)

Fig. 5. Closed trajectory test with four 90 degree angles. a) Start of the test. b) End of a trajectory.

In the test, the robot was programmed to travel the path several times continuously. The starting point of the test was marked with a circle, the starting point of each cycle with a point and the end point of each cycle with a cross (Fig. 6). In this way the accumulation of error throughout the test can be appreciated and measured. After forty 90-degree turns the accumulated error is $7.5 \mathrm{~cm}$ deviation from its point of origin. We also observed a slight deformation in the rectangle traced by its trajectory (Fig. 7). Table 1 summarizes the test results.

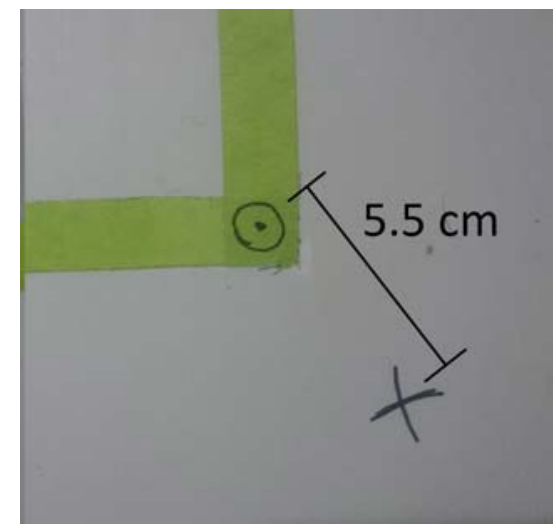

Fig. 6. Accumulated error after seven path cycles. 


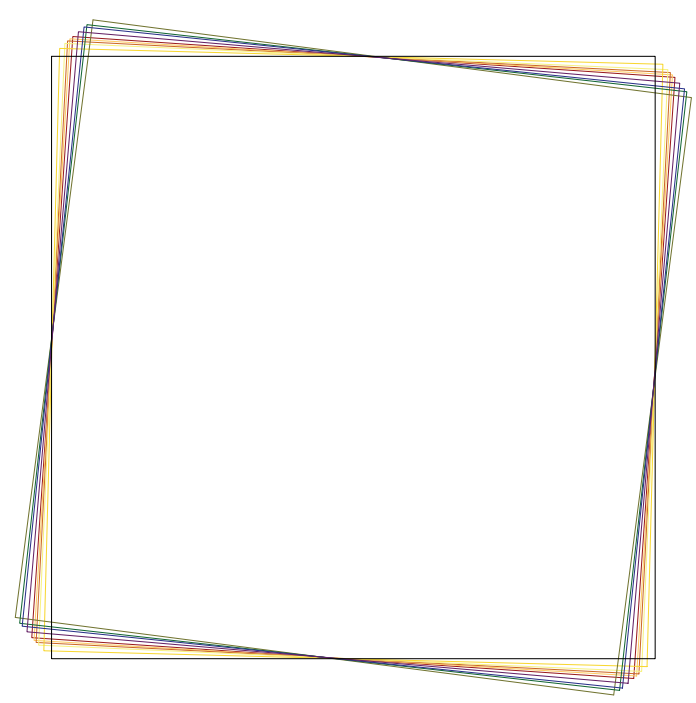

Fig. 7. Distortion of the trajectory during the test.

Table 1. Turning test results

\begin{tabular}{|c|c|c|}
\hline No & Accumulated error $(\mathrm{cm})$ & Deviation angle (degrees) \\
\hline 1 & 0 & 0 \\
\hline 2 & 1.4 & 1 \\
\hline 3 & 0.9 & 0.5 \\
\hline 4 & 1.4 & 1 \\
\hline 5 & 0.5 & 0.5 \\
\hline 6 & 0.6 & 0.4 \\
\hline 7 & 0.7 & 0.5 \\
\hline 8 & 0.7 & 1 \\
\hline 9 & 0.6 & 0.5 \\
\hline 10 & 0.7 & 1 \\
\hline Total & 7.5 & 6.4 \\
\hline
\end{tabular}

After several tests an average error per cycle of $0.73 \mathrm{~cm}$ and 0.61 degrees were established.

\section{B. Straight line advance tests}

For this test we define a straight path of two meters which should travel the robot in a straight line at constant speed (Fig. 8). This straight line serves as a reference to determine the deviation to the straight advance of the robot, measured in degrees. This type of error is mainly caused by small differences in the mechanical construction of the robot's lateral actuators. Table 2 summarizes the test result.).

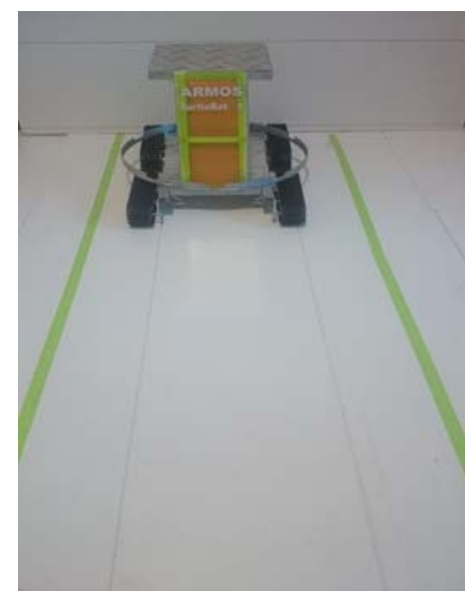

Fig. 8. Environment for straight line advance testing. 
Table 2. Straight line advance tests results

\begin{tabular}{|c|c|c|}
\hline No & Distance travelled $(\mathrm{m})$ & Deviation angle (degrees) \\
\hline 1 & 2.02 & 2.5 \\
\hline 2 & 2.00 & 1.0 \\
\hline 3 & 1.98 & 1.0 \\
\hline 4 & 2.01 & 3.5 \\
\hline 5 & 1.96 & 1.5 \\
\hline 6 & 2.01 & 6.0 \\
\hline 7 & 2.01 & 5.5 \\
\hline 8 & 2.01 & 3.0 \\
\hline 9 & 2.03 & 4.5 \\
\hline 10 & 2.00 & 1.0 \\
\hline Average & 2.00 & 2.95 \\
\hline
\end{tabular}

The distance travelled and accumulated error data shown in Table 2 again indicate that the error is small, predictable and can be easily corrected by some global positioning algorithm. This test was also performed several times, and the average error for the two meters remained close to three degrees (inclination to the left of the robot).Just like the first test, this constant, and therefore predictable, behaviour allows the error to be compensated by software, and reduces the use of a global positioner.

\section{CONCLUSION}

In this paper we present the derivation of the cinematic model of the ARMOS TurtleBot 1 robotic platform, and its performance was evaluated by odometric navigation tests. The ARMOS TurtleBot 1 robot has two DC motors on each side activated simultaneously on two crawler tracks. The characteristic of its movement was modeled as a differential wheeled robot. The differential wheeled robot has a kinematics described from the geometry of its structure and movement, which can be characterized by a set of differential equations with state variables defined by their speed (angular and linear) and angle on the plane of the ground. This model was identified from measurements on the ARMOS TurtleBot 1 robot to determine its model. The model was then evaluated through two tests: 90-degree turns and straight-line advances. In both cases the accumulative errors found were small, constant and predictable, so their adjustment can be incorporated into the robot model.

\section{ACKNOWLEDGMENT}

This work was supported by the Universidad Distrital Francisco José de Caldas, in part through CIDC, and partly by the FacultadTecnológica. The views expressed in this paper are not necessarily endorsed by Universidad Distrital. The authors thank the research group ARMOS for the evaluation carried out on prototypes of ideas and strategies.

\section{REFERENCES}

[1] H. Christensen, A. Okamura, M. Mataric, V. Kumar, G. Hager and H. Choset, Next generation robotics, Computers and Society, 2016 : pp. 3-21.

[2] K. Rajan and A. Saffiotti, Towards a science of integrated ai and robotics, Artificial Intelligence, vol. 247, 2017: pp. 1-9.

[3] E. Christoforou and A. Müller, R.U.R. revisited: Perspectives and reflections on modern robotics, International Journal of Social Robotics, vol. 8(2), 2016: pp. 237-246.

[4] V. Unhelkar, P. Lasota, Q. Tyroller, R. Buhai, L. Marceau, B. Deml and J. Shah, Human-aware robotic assistant for collaborative assembly: Integrating human motion prediction with planning in time, IEEE Robotics and Automation Letters, vol. 3(3), 2018: pp. 2394-2401.

[5] M. Carrozza, The socialization of robotics, The Robot and Us. Biosystems\&Biorobotics, vol. 20, 2019.

[6] R. Guzmán, R. Navarro, M. Cantero and J. Ariño, Robotnik - professional service robotics applications with ROS, Robot Operating System (ROS), vol. 707, 2017: pp. 419-447.

[7] Y. Sefidgar, K. MacLean, S. Yohanan, H. Loos and E. Crof, Design and evaluation of a touch-centered calming interaction with a social robot, IEEE Transactions on Affective Computing, vol. 7(2), 2016: pp. 108-121.

[8] A. Rendón, Evaluación de estrategia de navegaciónautónomabasada en comportamientoreactivo para plataformasrobóticasmóviles, Tekhnê, vol. 12(2), 2015: pp. 75-82, ISSN 1692-8407.

[9] M. Wada and R. Torii, Cooperative transportation of a single object by omnidirectional robots using potential method, 16th International Conference on Advanced Robotics (ICAR 2013), 2013, pp. 1-6.

[10] M. Jokesch, M. Bdiwi and J. Suchý, Integration of vision/force robot control for transporting different shaped/colored objects from moving circular conveyor, IEEE International Symposium on Robotic and Sensors Environments (ROSE 2014), 2014, pp. $78-82$.

[11] A. Jevtić, G. Doisy, Y. Parmet and Y. Edan, Comparison of interaction modalities for mobile indoor robot guidance: Direct physical interaction, person following, and pointing control, IEEE Transactions on Human-Machine Systems, vol. 45(6), 2015: pp. 653-663.

[12] J. Chandra and A. Prihatmanto, Stereo visual odometry system design on humanoid robot nao, 6th International Conference on System Engineering and Technology (ICSET 2016), 2016, pp. 34-38.

[13] X. Nan and X. Xiaowen, Robot experiment simulation and design based on festorobotino, IEEE 3rd International Conference on Communication Software and Networks, 2011, pp. 160-162.

[14] A. Melingui, T. Chettibi, R. Merzouki and J. Mbede, Adaptive navigation of an omni-drive autonomous mobile robot in unstructured dynamic environments, IEEE International Conference on Robotics and Biomimetics (ROBIO 2013), 2013, pp. 1924-1929. 
[15] U. Sychou, Control algorithm for mobile robots based on fixed-point chaotic sequences generator, The 10th International Conference on Digital Technologies, 2014, pp. 333-337.

[16] W. Peng, L. Bingqiang, Z. Zhiqiang, D. Qiang and Z. Chunxi, Design of navigation system for the inspection robot in unattended operation substation, Asia-Pacific Power and Energy Engineering Conference, 2010, pp. 1-4.

[17] M. Ibraheem, Gyroscope-enhanced dead reckoning localization system for an intelligent walker, International Conference on Information, Networking and Automation (ICINA 2010), 2010, pp. V1-67-V1-72.

[18] J. Borenstein, H. Everett and L. Feng, Navigating Mobile Robots: Systems and Techniques, A. K. Peters Ltd., 1996.

[19] J. Singh and P. Chouhan, A new approach for line following robot using radius of path curvature and differential drive kinematics, 6th International Conference on Computer Applications In Electrical Engineering-Recent Advances (CERA 2017), 2017, pp. 497-502.

\section{AUTHOR PROFILE}

Cristian FerneyPenagos Beltran. Electricity Technologist, Researcher at the SIEPOT research incubator attached to the ARMOS research group at the Universidad Distrital Francisco José de Caldas.

Luis Angel Pacheco Romero. Electricity Technologist, Researcher at the SIEPOT research incubator attached to the ARMOS research group at the Universidad Distrital Francisco José de Caldas.

Fredy Hernán Martínez Sarmiento. PhD in Engineering - Systems and Computation, Professor at the Universidad Distrital Francisco José de Caldas, Director and principal researcher of the ARMOS research group. 